\title{
Antimicrobial Colossolactones from a Nigerian Polypore Ganoderma colossum (Fr.) C.F. Baker
}

\author{
L. N. Ofodile, ${ }^{1,2}$ N. U. Uma, ${ }^{3}$ T. Kokubun,${ }^{2}$ R. J. Grayer, ${ }^{2}$ O. T. Ogundipe, ${ }^{3}$ \\ EंM. S.J. Simmonds ${ }^{2}$ \\ ${ }^{1}$ Department of Biological Sciences, Yaba College of Technology, P. M. B 2011, Yaba, Lagos, \\ Nigeria; ${ }^{2}$ Royal Botanic Gardens, Kew, Richmond, Surrey, TW9 3AB, UK; ${ }^{3}$ Department of \\ Botany/Microbiology, University of Lagos, Akoka, Lagos, Nigeria
}

A number of synthetic antimicrobial agents have been developed to kill microorganisms effectively. However, drug resistance often occurs when these agents are used long-term. As a result, there has been a tremendous interest in the antimicrobial properties of plants and fungi. Ganoderma species are widely used, especially in Asia, for the treatment of chronic diseases such as cancer, hepatitis, bronchitis, asthma, and haemorrhoids. Many publications have also described the antimicrobial properties of compounds isolated from various polypores, including Ganoderma species. Nigeria has a rich biota of polypores, but the antimicrobial activity of these fungi has not been reported previously.

The antimicrobial activity of a new colossolactone, 23-hydroxycolossolactone $\mathrm{E}$, and two known colossolactones isolated from Ganoderma colossum (Ganodermataceae) were tested against gram-posi- tive and gram-negative bacteria. Fruiting bodies of G. colossum were collected from a dead log of Delonix regia (Fabaceae), Yaba College of Technology, Lagos, Nigeria. The polypore was morphologically determined as $G$. colossum by the truncate, ornamental, yellow basidiospore that is $11-17 \times 7-11$ $\mathrm{cm}$ in diameter. A voucher specimen accession $\mathrm{K}$ (M) 120802 was deposited in the herbarium at the Royal Botanic Garden, Kew, UK. Bioassay guided fractionation of compounds from a fungal sample was carried out using various chromatographic techniques. A ground sample was extracted with 100 $\mathrm{mL}$ of $n$-hexane: dichloromethane. The residue was reconstituted in methanol and subjected to column chromatography. Seventeen fractions obtained were fractionated using analytical thin layer chromatography (TLC). Chromatograms were inspected under UV light at 254 and $366 \mathrm{~nm}$. 
The activities were evaluated by the thin layer chromatography agar overlay method. Activity against Bacillus subtilis and Pseudomonas syringae was detected at different zones on the TLC plates: $\mathrm{R}_{f} 0.10-0.70,0.80$, and 0.98 by fractions (4-12). The active compounds were detected by spraying with anisaldehyde/sulphuric acid. The combined fractions (4-12) were evaporated and chromatographed by preparative HPLC using $50 / 50 \%$ methanol in water, a linear gradient for 20 minutes, and having a flow rate of $4.5 \mathrm{~mL} / \mathrm{min}$. The compounds were eluted at 14.2 minutes $(1,5.0$ $\mathrm{mg}), 19.2$ minutes $(2,0.9 \mathrm{mg})$, and 15.7 minutes (3, $0.8 \mathrm{mg})$ in the preparative HPLC. ${ }^{1} \mathrm{H}$ and ${ }^{13} \mathrm{C}$ NMR spectra were recorded on a Bruker Advance $400 \mathrm{MHz}$ using tetramethylsilane (TMS) as an internal chemical shift reference for samples at $30^{\circ} \mathrm{C}$, with chemical shift values of $0.00 \mathrm{ppm}$ in both ${ }^{1} \mathrm{H}$ and ${ }^{13} \mathrm{C}$ NMR spectra.

All NMR data were recorded using $\mathrm{CDCL}_{3}$. The structures of compounds 1-3 were determined by ID ${ }^{1} \mathrm{H}$ selection NOESY and ${ }^{1} \mathrm{H}$ and ${ }^{13} \mathrm{C}$ NMR spectroscopy. The sequence of carbon and hydrogen atoms was settled by two-dimensional heteronuclear multiple bond correlation spectroscopy, and two-dimensional heteronuclear single quantum coherence spectroscopy (HSQC, HMBC). The compounds were stable. Compounds 1,2, and 3 (spotted on a TLC plate) were detected by deep green, violet, and deep green staining, respectively, with anisaldehyde in sulphuric acid. Compound 1 was identified as 23-hydroxylcolossolactone E. Compounds 2 and 3 were identified as colossolactone $\mathrm{B}$ and $\mathrm{E}$, respectively. Antimicrobial activity of compounds was performed with Gram-positive and Gram-negative bacteria by the thin layer chromatography agar overlay method. Aliquots $(1.6-8.0 \mu \mathrm{g})$ of compounds were spotted on the TLC plates and eluted with chloroform:methanol (9:1). Seeded medium $(50 \mathrm{~mL})$ was evenly spread on the TLC plates. Chloranphenicol was used as the positive control. Plates were incubated overnight at $37^{\circ} \mathrm{C}$, after which $\rho$-iodonitrotetrazolium violet solution was added. The results showed that colossolactone E and 23hydroxycolossolactone E were active against Bacillus subtilis and Pseudomonas syringae. Colossolactone B was not active against the bacteria. The activities of Colossolactone $\mathrm{E}$ and 23-hydroxylcolossolactone $\mathrm{E}$ were stronger against $B$. subtilis than $P$. syringae. The zones of inhibition of bacteria by 23-hydroxylcolossolactone $\mathrm{E}$ were found to be similar to those of chloramphenicol.

As yet, there is no publication on the use of isolates of Ganoderma from Nigeria in the treatment of infections, despite its well-documented medicinal uses in Asia. Nigeria is a country where immunesuppressive diseases, such as HIV, are endemic, and the availability of antibacterial products that could be sustainably harvested could be significant to poor rural communities. 